\title{
Smart Meters in Future Power Grid: A Review
}

\author{
M. Rajasree \\ Department of EEE, CVR College of Engineering, Hyderabad, India \\ Email:rajasreemantri@gmail.com
}

\begin{abstract}
Smart meter is the most important modern energy meter in smart grid. Apart from measuring energy consumption, it also provides additional information to the utility company and the consumers for effective energy management. Integration of smart meters into electricity grid involves implementation of various communication technologies and software depending on the features that the situation demands. This paper outlines some features and benefits of a smart meter, current status as well as various future projects of smart grid development in top five electricity generating countries worldwide. In addition, it introduces some basic communication technologies used in smart grid and the challenges of smart meters in the future.
\end{abstract}

Index Terms - Smart grid, Smart meter, Communication technologies, Advanced Metering Infrastructure (AMI).

\section{INTRODUCTION}

A traditional grid is an electrical system that supports operations like generation, transmission, distribution and utilization of electricity. The conventional power grids are generally used to carry power from a few central generators to a large number of consumers but there is no two-way communication that allowing interaction between end users and the grid. A smart grid is a modern electrical grid in which electricity and information flows in two-way to improve reliability, efficiency, sustainability and economics of the grid. By utilizing modern information technologies, the smart grid is capable of delivering power in more efficient ways and it could respond to events that occur anywhere in the grid. Table I shows a brief comparison between the existing grid and the smart grid [1].

One of the important way to modernize the present electric grid into a smart grid is the use of smart meters. It is a modern energy meter which gathers information from the users, load equipment and calculate the energy usage of consumers therefore provides additional information to the power company and / or the system operator.

A variety of sensors and control devices and supportive communications infrastructure are used in smart meters.

TABLE I

COMPARISON BETWEEN THE TRADITIONAL GRID AND THE SMART GRID

\begin{tabular}{|l|l|}
\hline Conventional grid & Smart grid \\
\hline communication is one-way & communication is two-way \\
\hline electromechanical & digital \\
\hline centralized generation & distributed generation \\
\hline limited control & pervasive control \\
\hline manual monitoring & self monitoring \\
\hline manual restoration & self-healing \\
\hline failures and blackouts & adaptive and islanding \\
\hline limited sensors & more sensors \\
\hline less customer choices & more customer choices \\
\hline
\end{tabular}

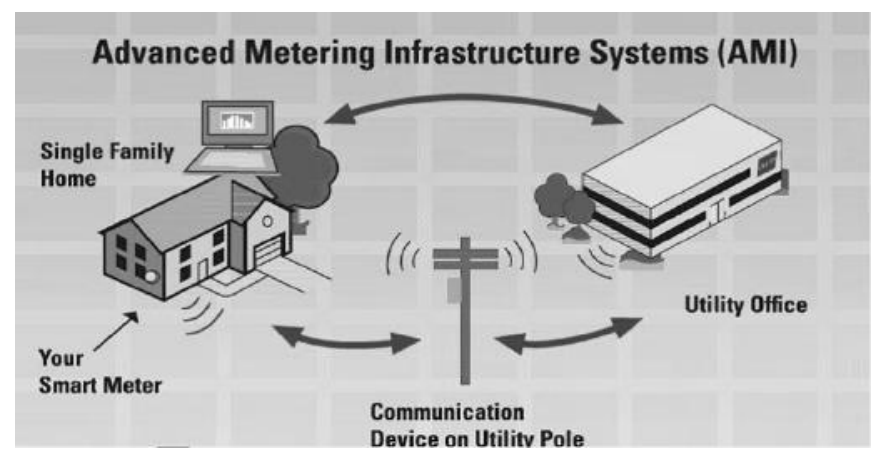

Figure 1. The Advanced Metering Infrastructure (AMI) System

AMI network is shown in figure 1 . It is in fact the collective term to describe the whole infrastructure that includes smart meters, two-way communication network and control center equipment. The control center equipment is connected to all the applications that gather and transfer energy usage information in real-time. AMI makes two-way communications with customers and is the backbone of smart grid. The objectives of AMI are remote meter reading with error free data, network problem identification, load profiling, energy audit and partial load curtailment in place of load shedding.

The remaining paper is scheduled as follows. section II describes smart meter design, functions and benefits; section III aims different countries government's present and future policies in brief; section IV outlines communication network and its related issues; section $\mathrm{V}$ explains the challenges of a smart meter and conclusions are drawn in Section VI .

\section{SMART METER}

A smart meter is usually an electronic device that records consumption of electric energy in intervals of an hour or less and enables real-time communication of energy usage data between customers and their utility companies for monitoring and billing purposes. The vast majority of these meters are installed at the residential level. In order to carry out a meter reading using a conventional meter, the meter reader needs to physically visit the customer premise and take the reading. This reading will be sent to the utility company for billing. But in case of smart meters this can be done automatically. The system operator will create a meter read request from the utility company office thus avoids manual intervention during meter reading and provides more accurate, real-time data to the utility company. Figure 2 shows the block diagrams of a traditional energy meter and a smart meter [2]. 
Conventional Energy Meter

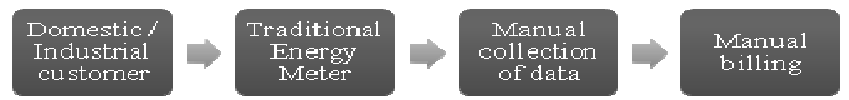

Smart Energy Meter

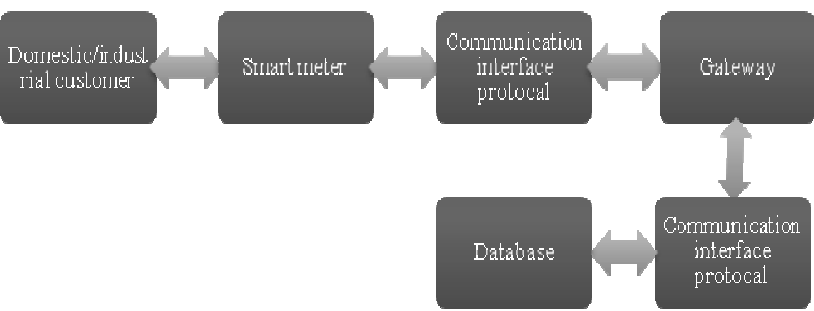

Figure. 2. The architectures of a conventional energy meter and a smart meter

A smart meter consists two units. One unit of the metering device is in the custody of the distribution or utility company and the other is the display unit which is at consumer's place. A smart meter has designed with built-intechnology to disconnect and reconnect certain loads remotely. Smart meters are implemented to monitor as well as to control end users and appliances to manage demand and load flow in the future. Smart meter's data comprises the unique meter identifier, data timestamp, the electricity utilized values and so on. Smart meters can gather diagnostic information about the distribution grid and home appliances and measures energy consumption from them to identify parameters and transfer the data to utilities and send back to the command signals in order to calculate the customer's bill and power consumption accordingly. Sometimes, a smart meter can also communicate with the other smart meter. Figure 3 shows the original model of a smart meter. Smart meters, which enable real-time communication of energy usage data between consumers and their utility companies, generate electric usage readings for every 15 minutes or one hour.

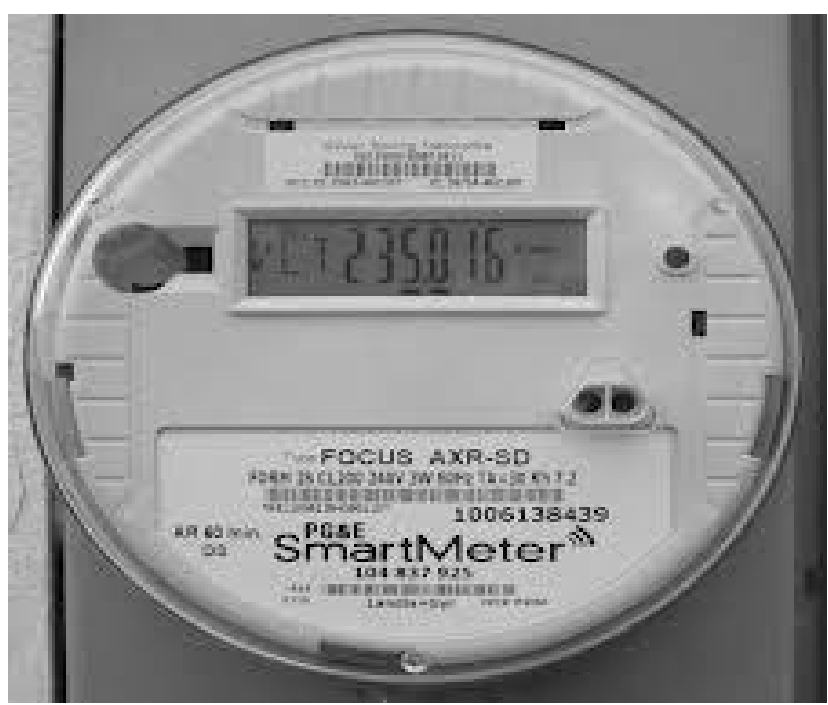

Figure 3. An actual model of a smart meter [3]

\section{A. The main functions of a smart meter}

Generally, smart meters are expected to have the following features like:

1. Two-way communication

2. Data collecting, recording and storing

3. Load control function

4. Programming function

5. Security function

6. Display function

7. Billing function

\section{B. Advantages of a smart meter}

The benefits of installing smart meters are numerous for many different stakeholders in different aspects of the smart grid system [4].

\section{Smart metering advantages to consumers:}

- It gives the customer more accurate and timely electrical billing.

- Consumers are able to estimate bills from the collected information of smart meters thus manage their energy consumption to reduce their electric bills.

- It helps the customer in a better way to use the electrical equipment during the expensive hours. Time of Use (ToU) tariff will offer choice to consumers to reduce the electricity consumption during peak hours which will bring considerable savings in their bills.

- It facilitates customers to switch/delay their electrical equipment to less expensive hours. For e.g. it offers homeowners and business owners a new level of energy intelligence with near real-time energy use data.

- There exists a high speed communication system between utility and customer.

- The smart meter will help in a faster restoration of the grid in case of faults or disturbances.

Smart Metering advantages to utilities:

- Lot of money can be saved by improving the remote area reading and billing system.

- Utility can better manage during peak load times.

- It makes more efficient use of energy and grid resources. Smarter systems will help integration of renewable energy to the grid and by deploying smart micro grids and encouraging distributed generation; power will be accessible to the remote areas as well.

- It offers a new tariff model in the electricity market.

- It improves the transformer load management for the transmission line.

- Utilities can control the power supplied to consumers.

- The accuracy of smart meter enables enhanced monitoring of system resources, which detects and mitigates energy threats on the grid by cyber-terrorist.

- $\quad$ AMI systems that track energy usage will help monitor power almost in real time thus leading to increased system transparency thus prevents loss due to electricity theft.

- Smart meters can also be used to shut off service to households and commercial establishments that don't pay their bills. 
- Using smart meters, the companies can limit the maximum electricity consumption and tries to encourage users to reduce their demands in the periods of peak load.

- The data collected by smart meters is used by utility to realize real-time pricing.

- Utility company can terminate or re-connect electricity supply to any consumer with a proper mechanism remotely in order to optimize the power flows according to the information sent from demand side.

Smart Metering advantages to Governments:

- It stimulates the economy by investing in smart metering networks.

- By increasing the awareness of consumption pattern, there will be a reduction in electricity consumption.

- Better load forecasting can be obtained for power grid and it will prevent large-scale black outs.

- The data given by smart meters helps in improving efficiency and reliability of service.

\section{PRESENT CONDITION IN TOP FIVE ELECTRICITY GENERATING COUNTRIES}

\section{A. China}

The advancing smart grid construction in China spurred the constant growth of smart meter demand. A primary element of this transformation is the deployment of interactive technologies, including smart meters for residences and businesses. The installed smart meters in China will reach 377 million by 2020 , growing from 139 million in 2012. The emerging rate for smart meters will reach 74 percent in 2013 and the figure is expected to hit 500 million in 2015 . The main reasons for this progress are [5]:

- The main force behind the efforts to construct the country's smart grid is the State Grid Corporation of China (SGCC). It is the government-owned electric utility that leads the market by a large margin

- China's metering program initially had the goal of installing smart meters in 95 percent of household by 2015, but the end date has been pushed forward to 2017 due to some reasons.

- Profiles are provided more than 40 main industry players, classified by different industries, along with forecasts for smart grid revenue and smart meter shipments through 2020.

- Tenders were invited by SGCC for smart meters as early as 2009 and as of September 2014, 21 tenders for a total of 310 million smart meters were invited, in which 3 tenders for 61.877 million smart meters were completed during Jan-Sept. 2014.

B. USA

The number of installed smart meters in the US grew by 33\% between May 2012 and July 2013. Figure 4 illustrates the historic and estimated path of smart meters installation in the US through 2015. This represents approximately
675,000 smart meters installed per month between now and the end of 2015 [6].

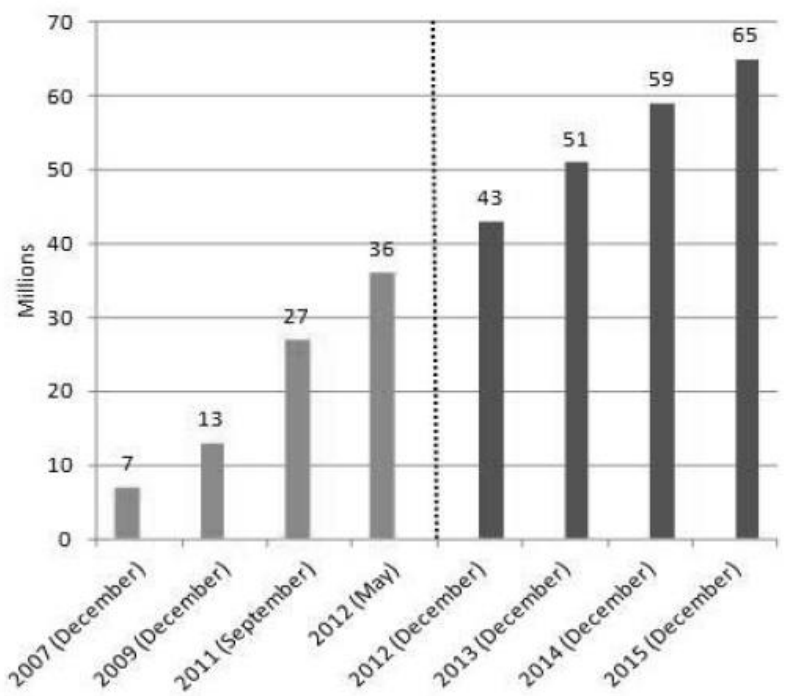

Figure .4, smart meter installations in the US: 2007-2015 (millions)

Some other policies regarding smart meters are:

- As of May 2012, Institute of Electrical Engineers (IEE) found that almost one-in three households now have a smart meter. By mid- decade, more than half the households in the country will have a smart meter [7].

- Based on submitted and approved AMI business plans, responses to survey questions and other public information, IEE finds that as of May 2012, 36 million smart meters have been installed and IEE estimates that approximately 65 million smart meters will be deployed by 2015 .

- The electric power sector is providing benefits to consumers through information gathered by smart meters. By the end of 2012, 22 utilities in 16 states will have smart meters fully deployed to their entire consumers, representing 29 million consumers.

- Millions of utility customers already have their energy usage data recorded by smart meters and the industry is working to translate the detailed smart meter data into customer-friendly information.

\section{C.Russia}

Russia is pursuing the state policy of innovation activity in the electricity sector. This applies to energy efficiency, renewable energy and smart grids. The energy strategy of Russia for the period up to 2030 aims at ensuring high energy, economic and environmental efficiency in the production, transport, distribution and demand of electricity. Russia has recorded a major reduction in losses and an increase in capacity to supply more power to customers since implementing a smart metering system [8].

Some National policies on smart meters are, such as:

- Smart meters and accounting systems should be installed at all participants of electricity market and thermal power in power plants and substations, in enterprises, and with the year 2012 in accordance with 
the Federal law No. 261-FZ dated 23.11.2009. The U.S. Company, in a partnership with Perm Energo - a division of the MRSK group and one of Russia's largest utility providers, was awarded a contract to network 10,000 homes as part of a 50,000 home test project that will be monitored by the Russian government for potential rollout across the country.

- Russia wants to reduce energy intensity - a measure of usage by 40 percent by 2020 to modernize Soviet-era companies.

- Echelon - which operates in Russia in partnership with local firm Energo Audit Control (EAC) said it should sell 200,000 meters in Russia next year, up from 100,000 in 2012.

\section{D .Japan}

Tokyo Electric Power Company (TEPCO)'s 27-million unit smart meter project is not just about smart meters but also about creating an end-to-end, citywide energy management platform meant to link meters, utility enterprise platforms and smart devices in homes and businesses [9]. TEPCO and its fellow utilities recently saw their smart meter deployment plants put on an increasingly fast track in hopes of managing the country's ongoing post-Fukushima energy crisis, as well as to prepare for a potential shift to a deregulated energy market in 2016.

Here's how this project is breaking new ground.

TEPCO wants all three flavors of meter network not just for meters, but for devices in homes and businesses. Toshiba , Landies and Gyy's work on the technology needed to meet TEPCO's multi-model metering communications plans involves multiple meter manufacturers, all building to a modular design that can permit the swapping out of three flavors of communication.

TEPCO needs its smart meter network to scale big and scale both ways. TEPCO's 27 million smart meters are each meant to network with multiple in-premise devices with scalability from the individual components back to the headend, to deal with those huge volumes of meter data.

\section{E. India}

India operates the world's largest synchronous grid covering an area of 3.28 million square kilometers with a connected capacity of $235 \mathrm{GW}$ and about 200 million customers. Even though 79 million households in India do not have access to electricity, but it's per capita consumption is one fourth of world's average[10].

Smart grid vision for India is "Transform the Indian power sector into a secure, adaptive, sustainable and digitally enabled ecosystem that provides reliable and quality energy for all with active participation of stakeholders"

In 2013, Government of India (GoI) issued "Smart Grid Vision and Roadmap for India" which is a 15 year roadmap for transformation of Indian power system to smart grids. With the recent activities around 14 smart grid pilot projects in different states, India has emerged as the hot destination for smart grids. India Smart Grid Forum (ISGF) is a public private partnership initiative of Ministry of Power (MoP), for accelerated development of smart grid technologies in the Indian power sector. ISGF was setup in
2010 to provide a mechanism through which academia, industry, utilities and other stakeholders could participate in the development of Indian smart grid systems and provide relevant inputs to the government's decision-making.

ISGF has 10 Working groups. They are:

1. Advanced Transmission

2. Advanced Distribution

3. Communications

4. Metering

5. Load Control

6. Regulatory \& Policy

7. Architecture \& Design

8. Pilots and Business Models

9. Cyber Security: newly formed

10. Renewables \& Micro grids: newly formed

The metering group aims are to assess the metering scenario in India and also to identify it's requirements and goals. The group 4 also focus on the available technologies and the best practices as well as identifying the gaps in existing solutions from an Indian perspective. It is worth mentioning that for Restructured Accelerated Power Development and Reforms Program (R-APDRP) metering applications the IEC 62056 It is worth mentioning that for Restructured Accelerated Power Development and Reforms Program (R-APDRP) metering applications the International Electrotechnical Commission (IEC) 62056 is chosen as the standard metering protocol. Central Power Research Institute (CPRI) has the test facility for carrying out conformance test for this protocol standard. The metering group can extend the additional design and standards required for smart meters. Metering group scope is such as:

- Study of country wide metering and billing practices. This includes Automatic Meter Reading (AMR) projects, merits \& demerits, understanding of present technology, regulatory provisions, Electricity Act (EA) and requirements for AMI infrastructure.

- To design different smart meters for single-phase and three- phase customers.

- To analyze different communication technologies. Information requirements.

- To analyze different information and interface device requirements.

- Meter Data Management Systems (MDMS).

India Smart Grid Task Force (ISGTF), launched along with ISGF is an inter-ministerial government task force. ISGTF mooted the idea of promoting 8 smart grid pilots in the country in different distribution utilities and it has 5 working groups, such as:

1. Pilots on New Technologies

2. Loss Reduction \& Analysis

3. Power to Rural/Urban Areas

4. Distributed Generation \& Renewables

5. Cyber Security

Smart Grid Vision and Road map for India has planned as, the $12^{\text {th }}$ five year plan (2012-17) road map of smart grid will meet the expectations of customers, the $13^{\text {th }}$ five year plan (2017-22) of smart grid vision for India will 
meet the requirements of utilities and the $14^{\text {th }}$ five year plan (2022-27) will hit government and regulators expectations.

\section{THE INFORMATION AND COMMUNICATION TECHNOLOGIES}

The communication medium in an AMI system must ensure the communication between the smart meters and the central computer at the service provider. The communication structure can be wired like Power Line Carrier (PLC) or wireless like Global System Mobile (GSM), ZigBee, Radio Frequency (RF) and Wide Area Measurement System (WAMS). The chosen way must take into account the distance between the devices and existing infrastructure [11].

The following are the factors that impact the selection of communication technology [12]. :

- Evaluation of the existing infrastructure.

- Impact on legacy equipment, functionality, technical requirements as well as the economic impact to the utilities and consumers.

\section{A. Power Line Carrier Communication (PLCC)}

PLCC is an approach to utilize the existing power lines for the transmission of information. In today's world every house and building has properly installed electricity lines. By using the existing AC power lines as a medium to transfer the information, it becomes easy to connect the houses with a high speed network access point without installing new wings. The data collected by smart meter can be transmitted to utility central collection point by using the utility power lines. Then the delivered data is further processed and analyzed. [11]. PLCC technology has some advantages like, it can improve cost effectiveness for rural lines, and make it possible to work for the remote area or over long distances. This technology also has some drawbacks like, it has longer data transmitting time than wireless, higher cost in urban areas and less bandwidth.

Some research has been conducted in the PLC area . Rakesh Rao [13] presented a method for identifying outlines among a set of smart meters by measuring the PLC signal strength between the communication node (transmitter) and residential smart meters. The PLC signal is used as a proactively avert local power outages. Mojtaba Rafiei [14] propose a practical smart metering approach which can be used for both type of AMR and AMI by using combination of PLC and Wi-Fi protocols. Liang Dong [15] present the noise characteristic and transmission characteristic of the power line channel at first, then establish the basic power line channel model according to measured data.

\section{B. Radio Frequency (RF)}

The collected data from end users is transmitted to data collector through wireless radio frequency using smart meters. Then, the data is processed and delivered in several methods to utility data systems at a central collection location. The utility billing, outage management and other systems use these data for operational and business purposes. The RF technologies are classified in to two. They are:

\section{Mesh Technology:}

The smart meters talk to each other to form a Local Area Network (LAN) cloud at the collection point. The collector transmits the data using different Wide Area Network (WAN) methods to the utility central location [11].

1. The mesh RF technology has some advantages, such as large bandwidth, acceptable latency and a typical operational frequency of $915 \mathrm{MHz}$.

2. The mesh RF technology also has some drawbacks, such as proprietary communication, the topography and long distance issues for the remote areas.

\section{Point to Point Technology:}

In this technology, smart meters talk directly to a collector, usually a tower. The tower collector transmits the data using various methods to the utility central location for processing [11].

1. Point to Point RF technology has some advantages, such as large bandwidth, little or no latency, direct communication with each endpoint, better throughput, and can cover longer distances.

2. Point to Point RF technology also has some disadvantages, such as the topography, long distance issues for remote areas, proprietary communications and less interface with Distribution Automation (DA) devices.

\section{Cellular networks}

Public cellular networks use in smart grid is slowly gaining a acceptance and acceleration across the world. Cellular network is now commonly seen as an additional connectivity option just like PLCC and RF mesh. Global System for Mobile communication (GSM) is a digital mobile telephony system that digitizes and compresses data before sending it. The main advantage of the GSM is its widespread use throughout the world and the use of Subscriber Identity Module (SIM) cards to send Short Message Service (SMS). [16].

\section{ZigBee}

ZigBee is a low-cost, low-power, wireless mesh networking standard. It is best suited for local coverage such as Home Area Networks (HANs). Smart grid considers ZigBee as a main communication as it controls the appliances automatically. ZigBee installation and upgrade cost is low, in addition it offers meter-to-meter communication and remote monitoring ability of whole home conditions [17].

Smart energy meters using both ZigBee and GSM technologies should have a transmitter and a receiver with both technologies. The meter can read the energy and send it to the receiver using GSM or ZigBee. The data management system collects and stores the data and uploads it to the internet. So, the consumer can check his information from the internet using a developed android program or through a website portal. The receiver can also send the consumption information to the user by an SMS message through the GSM network [16]. 


\section{E. Web Access Management System (WAMS)}

WAMS is a solution for obtaining accurately time synchronized measurements of electrical parameters of a power grid, spanning over wide geographical areas such as large regions and even entire nations. The analysis of WAMS data provides a real-time view of the grid condition, allows certain situations to be predicted and preventative action taken to avoid large scale outages or blackouts. WAMS enhances the the functionality of traditional operation technology such as Supervisory Control And Data Acquisition (SCADA) systems by collecting more data from devices called Phasor Measurement Units (PMU) situated at strategic positions with in the grid [10].

A typical SCADA system measures the magnitude of electrical parameters such as voltage, current, frequency and power at a rate of 1 sample per second but WAMS measures both the magnitude and phase angle of electrical parameters at about 30 to 120 samples per second with accurate synchronized time-stamping. This feature enables WAMS to provide a real-time view of grid conditions. More data, faster access to data and real-time analysis of data provides situational awareness to grid operators, enabling them to take intelligence based and timely control actions to preserve the grid's stability and efficiency.

The key is that WAMS provides much more information with higher resolution and clarity, as compared to traditional operational technology.

\section{THE CHALLENGES OF SMART METER}

Despite its widespread benefits, deploying smart meters presents three major challenges that include:

1. High Capital Cost: A full scale deployment of AMI requires expenditure on all hardware and software components, smart meters, network infrastructure and network management software along with cost assosiated with the installation and maintenance of meters and information technology systems.

2. integration: AMI is a complex system of technologies that must be integrated with utilitie's information technology system, including Customer Information Systems (CIS), Geographical Information Systems (GIS), Outage Management Systems (OMS), Work Management Systems (WMS), Mobile Workforce Managemet (MWM), SCADA sytem, Distribution Automation System (DAS) etc.

3. Standardization: Interoperability standards to be defined, which set uniform requirements for AMI technology, deployment and general oprations are the keys to successfully connecting and maintaining an AMI based grid system.

\section{CONCLUSIONS}

This paper reviews several important aspects of smart metering. It presents the advantages of a smart meter system from the point of view of consumers, utilities and governments respectively. In addition, it presents the current situation and future objectives of leading countries in terms of electricity generation. Moreover, research related to different communication technologies is presented in detail. Finally, the paper arises various challenges to be met by the smart meter.

\section{REFERENCES}

[1] Fang Xi, Misra Satyajayant, Xue Guoliang, Yang Dejun, "Smart Grid -The New and Improved Power Grid: A Survey," Communications Surveys \& Tutorials, IEEE, vol. 14, issue.4, pp. 6-9, 2012.

[2] Lingfeng Wang, Devabhaktuni, V., Gudi, N., "Smart Meters for Power Grid - Challenges, Issues, Advantages and Status," 2011 IEEE/PES Power Systems Conference and Exposition (PSCE), pp. 1-7, March 2011.

[3] "Smart meter", Available: http://sjvceonews.blogspot.in

[4] Jixuan Zheng, Li Lin, cnDavid Wenzhong Gao, "Smart Meters in Smart Grid: An Overview "IEEE Green Technologies conference 2013.

[5] "Smart meter projects in china", http://www.sgcc.com.cn/ywlm/ index.shtml .

[6] "Samrt meter projects in USA" www.edisonfoundation.net/IEE.

[7] C. Bennett and D. Highfill "Networking AMI Smart Meters" in Proc. Energy 2030 Conference iEEE Atlanta, GA 17-18 pp. 1 - 8 , November 2008.

[8] Wiebe Mulder, Aleksey Nikolaev, Alexander Osadchiev, Svetlana Kleeva "Market scan smart meters and smart grid in Russia" Amhem,15 March 2013.

[9] "Japan Smart meter projects" www.tepco.co.jp/en/indexe.html

[10] "Smart meter projects in India" www.indiasmartgridforum.com

[11] "Smart Meters and Smart Meter Systems:A Metering IndustryPerspective,"Available: http://www.aeic.org/meter_service/smartmetersfinal032511. pdf

[12] Parag Kulkarni, Sedat Gormus, Zhong Fan, and Benjamin Motz ,"A Mesh-Radio-Based Solution for Smart Metering Networks," Communications Magazine, IEEE, vol. 50, issue. 7, pp. 86-95, July 2012.

[13] Rakesh Rao, Srinivas Akella, Gokhan Guley, Power line carrier (PLC) signal analysis of smart meters for outlier detection," 2011 IEEE International Conference on Smart Grid Communications (SmartGridComm), pp. 291-296, October 2011.

[14] Mojtaba Rafiei, S.Mehdi Eftekhari, "A practical smart metering using combination of power line communication (PLC) and WiFi protocols," 2012 Proceedings of 17th Conference on Electrical Power Distribution Networks (EPDC), pp. 1-5, May 2012.

[15] Liang Dong, Zhang BaoHui, "Design and Emulation of highspeed narrowband PLC system for Smart meter reading," 2010 Asia-Pacific on Power and Energy Engineering Conference (APPEEC), pp. 1-4, March 2012.

[16] Anmar Arif*, Muhannad AI-Hussain, Nawaf AI-Mutairi, Essam AI-Ammar Yasin Khan and Nazar Malik "Experimental Study and Design of Smart Energy Meter for the Smart Grid" IEEE 2013.

[17] J. Hurwitz and K. Wing-Hung"ES6: Technologies for smart grid and smart meter," Solid-State Circuits Conference Digest Technical Papers (ISSCC), IEEE international, pp. 533, 20-24 February 2011. 